\title{
Flow and release optimization in manufacturing systems represented as timed event graphs
}

\author{
A. Di Febbraro, R. Minciardi, M. Profumo, and S. Sacone \\ Department of Communication, Computer, and System Sciences, \\ University of Genova, Via Opera Pia 13, I-16145 Genova, Italy. \\ Tel: +39-10-3532748. Fax: +39-10-3532948. \\ e-mail: angelaedist. unige.it, riccardoedist.unige.it
}

\begin{abstract}
Timed Event Graphs can be applied successfully to the modelling, the analysis and the optimization of manufacturing systems. The choice of using such a particular class of Petri Nets allows to apply a set of related algebraic results to determine some performance measures as functions of a set of decision parameters. On this basis, performance optimization problems can be posed and solved as mathematical programming problems. In this paper, the problem consisting in the maximization of the weighted system throughput, while keeping the steady-state flow time of each product limited by an a-priori upper bound, is considered. This problem turns out to be a linear fractional programming one, which can be solved by means of standard techniques and software tools.
\end{abstract}

Manufacturing systems, Petri Nets, optimization.

\section{Keywords}

\section{INTRODUCTION}

A special class of Petri Nets, the Timed Event Graphs (or Timed Marked Graphs), has been investigated in the last years, as regards the development and the application of mathematical tools for the performance analysis. In particular, in (Cohen et al., 1985; 1989; Baccelli et al., 1992) a powerful set of algebraic results which allow a complete mathematical analysis of Timed Event Graphs have been provided. The main purpose of this paper is that of showing how such results can be applied to evaluate performance indices of interest, for a manufacturing system, as functions of a set of decision parameters. In this way, it is made possible to state and solve performance optimization problems through the application of proper mathematical programming techniques.

To this end, it is necessary to represent the manufacturing system model as a Timed Event Graph, that is, as a Timed Petri Net where each place has exactly one input and one output 
arc. This somehow limits the generality of the manufacturing system model to be considered, yet it is possible to treat significant models and optimization problems.

\section{THE MODEL OF THE MANUFACTURING SYSTEM AND ITS REPRESENTATION AS A TIMED EVENT GRAPH}

\subsection{General characteristics of the model}

A model of a manufacturing system is considered with $\mathrm{p}$ different classes of products, $\mathbf{P}_{\mathbf{l}}, \ldots, \mathbf{P}_{\mathbf{p}}$. Starting from basic components, each product of class $\mathrm{P}_{\mathrm{i}}, \mathrm{i}=1, \ldots, \mathrm{p}$, is obtained through a given set of operations o(i,1) ..o o(i, $\left.n_{i}\right)$, structured in an in-tree, where $o\left(i, n_{i}\right)$ is the final operation, and each join corresponds to an assembly operation. Operations can be simple, i.e., requiring one part as an input and giving one part as an output, or assembly ones. It is assumed that: a) no assembly operation requires as input two or more identical parts; b) when a basic component enters the system it is immediately assigned to a certain class of products; $c$ ) there may be a minimum waiting time to be observed between any pair of subsequent operations.

A (physical) function (e.g., painting, welding) $\phi=f(o(i, j))$ is associated with each operation $o(i, j), i=1, \ldots, p, j=1, \ldots, n_{i}$. Each operation $o(i, j), i=1, \ldots, p, j=1, \ldots, n_{i}$, can be executed by any machine $M_{k}, k \in M(i, j)$, where $M(i, j)$ is defined as the set of indices of machines that can accomplish the function $\mathrm{f}(\mathrm{o}(\mathrm{i}, \mathrm{j}))$. Machines are never off and no preemption is allowed.

It is assumed that the set of $\mathrm{m}$ machines, $\mathrm{M}_{1}, \ldots, \mathrm{M}_{\mathrm{m}}$, is partitioned into a set $\mathrm{BM}$ of batch machines, and a set $\overline{\mathrm{BM}}$ of ordinary machines. A batch machine can execute a batch operation, i.e., an operation resulting from the simultaneous execution of (simple) operations requiring the same function (e.g., heating, washing). Each batch machine can perform only one function. No function, hence no operation, implemented by a batch machine can be executed by any machine in $\overline{\mathrm{BM}}$. Each machine $\mathrm{M}_{\mathrm{k}} \in \overline{\mathrm{BM}}$ includes $\mathrm{s}_{\mathrm{k}}$ independent equivalent servers, $\mathrm{s}_{\mathrm{k}} \& 1$, each of which can perform a single operation at a time. A fixed set-up time may be necessary between the executions, on the same server of a machine in $\overline{\mathrm{BM}}$, of two operations requiring different functions.

The presence of set-up times, as well as the fact that the elementary operations needed to mianufacture products could not be so relevant to be considered individually, makes it convenient to group the single operations performed by machines in $\overline{\mathrm{BM}}$ in macrooperations. If $t(i, j, k)$ is the time (deterministic and known) required by machine $\mathrm{M}_{\mathbf{k}}$ to perform $o(i, j)$, then $T(i, j, k)=t(i, j, k) \cdot N(i, j, k)$ is the time required to perform the corresponding macrooperation $\mathrm{O}(\mathrm{i}, \mathrm{j}, \mathrm{k})$ on a lot of dimension $\mathrm{N}(\mathrm{i}, \mathrm{j}, \mathrm{k})$. In the case of an assembly macro-operation $O(i, j, k), N(i, j, k)$ not only represents the size of the lots of assembled parts, but also that of the lots of the parts to be assembled.

As regards batch operations, the lot sizes do not affect the execution times, but are to be taken into account in order to ensure the fulfilment of 'capacity' constraints affecting such operations. In general, there is a number of batch operations implemented by a machine $M_{h} \in B M$, say $B O(h, 1), \ldots, B O\left(h, b_{h}\right)$. Each one of such operations arises from the simultaneous execution of a set of operations on a set of corresponding lots of parts. Let $\Lambda[\mathrm{BO}(\mathrm{h}, \mathrm{r})]$ the set of operations corresponding to the batch operation $\mathrm{BO}(\mathrm{h}, \mathrm{r})$. Then, the following constraint must be satisfied

$$
v_{\text {min }}^{h, r} \sum_{o(i, j) \in \Lambda[B O(h, r)]} N(i, j, h), v_{\max }^{h, r} \quad \forall M_{h} \in B M, j=1, . ., n_{i}, r=1, . ., b_{h}
$$


where $v_{\min }^{h, r}\left(v_{\max }^{h, r}\right)$ is an a-priori given lower (upper) bound. The time required to implement any batch operation $B O(h, r)$ is TB(h), independent of $r$ and of the lot sizes $N(i, j, k)$ of the parts involved. The sets $\Lambda(B O(h, r)), M_{h} \in B M, r=1, ., b_{h}$, defining the batch operations for machine $\mathrm{M}_{\mathrm{h}}$, are supposed to be a-priori given.

It is assumed that for each kind of product $i$, the lots of all the relevant basic components are released synchronously and at a constant rate $1 / \pi(i)$. The time instant in which the first lot(s) of basic component(s) is (are) released is denoted by $\tau(\mathrm{i})$, with $\tau(\mathrm{i}) \geq 0 \forall \mathrm{j}=1, \ldots, \mathrm{p}$. The number of lots to be released is infinite.

The complete definition of the system model requires to specify the system behaviour whenever alternatives are possible as regards: i) how to assign operations to machines; ii) how to sequence macro-operations, or batch operations, assigned to the same machine. As regards the first issue, the execution of operation o(i,j) is shared out among machines $\mathrm{M}_{\mathrm{k}}, \mathrm{k} \in M(\mathrm{i}, \mathrm{j})$, by specifying the sizes of the lots $N(\mathrm{i}, \mathrm{j}, \mathrm{k}), \mathrm{k} \in M(\mathrm{i}, \mathrm{j})$, which are among the decision variables of the problem. As for the operation sequencing, it is assumed that, for each server of machine $\mathrm{M}_{\mathrm{k}}$ in $\overline{\mathrm{BM}}$, and for each machine $\mathrm{M}_{\mathrm{k}}$ in $\mathrm{BM}$, a fixed (known) service sequence $S_{\mathrm{k}}$ of macroor batch operations is applied. The set of decision variables affecting the considered model can be represented as the collection of the components of vector $\xi=\operatorname{col}[N(\mathrm{i}, \mathrm{j}, \mathrm{k}), \pi(\mathrm{i}), \tau(\mathrm{i})$, $\left.\mathrm{i}=1, \ldots, \mathrm{p} ; \mathrm{j}=1, \ldots, \mathrm{n}_{\mathrm{i}} ; \mathrm{k} \in M(\mathrm{i}, \mathrm{j})\right]$. Instead, the initial marking of the net is considered to be fixed.

\subsection{An example}

In order to show that the above general model admits a representation as a Timed Event Graph, it is worth considering a specific example, namely a manufacturing system with two classes of products, $P_{1}$ and $P_{2}$, and five machines $M_{1}, . ., M_{5}$, where $M_{5}$ is a batch machine, performing only one batch operation. Machines $M_{1}$ and $M_{2}$ have two servers, whereas $M_{3}$ and $\mathrm{M}_{4}$ are monoserver. Products of class $\mathrm{P}_{1}$ and $\mathrm{P}_{2}$ are obtained through a processing sequence of four simple operations $[o(i, 1), o(i, 2), o(i, 3), o(i, 4)], i=1,2$. The batch operation $\mathrm{BO}(5,1)$ is made up of operations $\mathrm{o}(1,3)$ and $\mathrm{o}(2,3)$. The set of indices of machines that can execute operations not involved in the batch operation are:

$$
\begin{aligned}
& M(1,1)=\{1,2\} \quad ; M(1,2)=\{1\} ; M(1,4)=\{4\} ; \\
& M(2,1)=\{2\} \quad ; \quad M(2,2)=\{3\} ; M(2,4)=\{3,4\} .
\end{aligned}
$$

The structure of the Timed Event Graph representing this manufacturing system is given in Figure 1. In this example, it is supposed that, for any service sequence, changing from an operation to another one implies also a change in the function implemented, so that a set-up time is required. Moreover, some nonzero minimum waiting times have to be fulfilled, namely between $o(1,2)$ and $o(1,3)$, between $o(2,1)$ and $o(2,2)$, and between $o(2,2)$ and $o(2,3)$.

Transitions are immediate, whereas nonzero token holding times are associated with some places. Their values are represented by the numbers of vertical bars in the places. Of course, the system is assumed to work under the Early Operational Mode (i. e., transitions fire as early as allowed). The notation $x_{i} / x_{k}$ is used to denote the place which has $x_{k}$ as input transition and $x_{i}$ as output transition. In the figure, places represented by thick black circles stand for macro- or batch operation executions. Only the holding times of places corresponding to macro-operations are affected by the choice of the lot sizes. A second class of places, the grey circles, has fixed holding times and represent minimum waiting times $\left(\mathrm{x}_{9} / \mathrm{x}_{8}, \mathrm{x}_{11} / \mathrm{x}_{6}, \mathrm{x}_{11} / \mathrm{x}_{10}\right)$, set-up times $\left(\mathrm{x}_{1} / \mathrm{x}_{6}, \mathrm{x}_{2} / \mathrm{x}_{8}, \mathrm{x}_{7} / \mathrm{x}_{4}, \mathrm{x}_{18} / \mathrm{x}_{10}, \mathrm{x}_{9} / \mathrm{x}_{20}, \mathrm{x}_{15} / \mathrm{x}_{19}, \mathrm{x}_{17} / \mathrm{x}_{16}\right)$, and lot decomposition/recomposition times $\left(\mathrm{x}_{14} / \mathrm{x}_{13}, \mathrm{x}_{12} / \mathrm{x}_{11}\right)$. The remaining places model conditions representing the availability of machines and lots, and have zero holding times, too. 


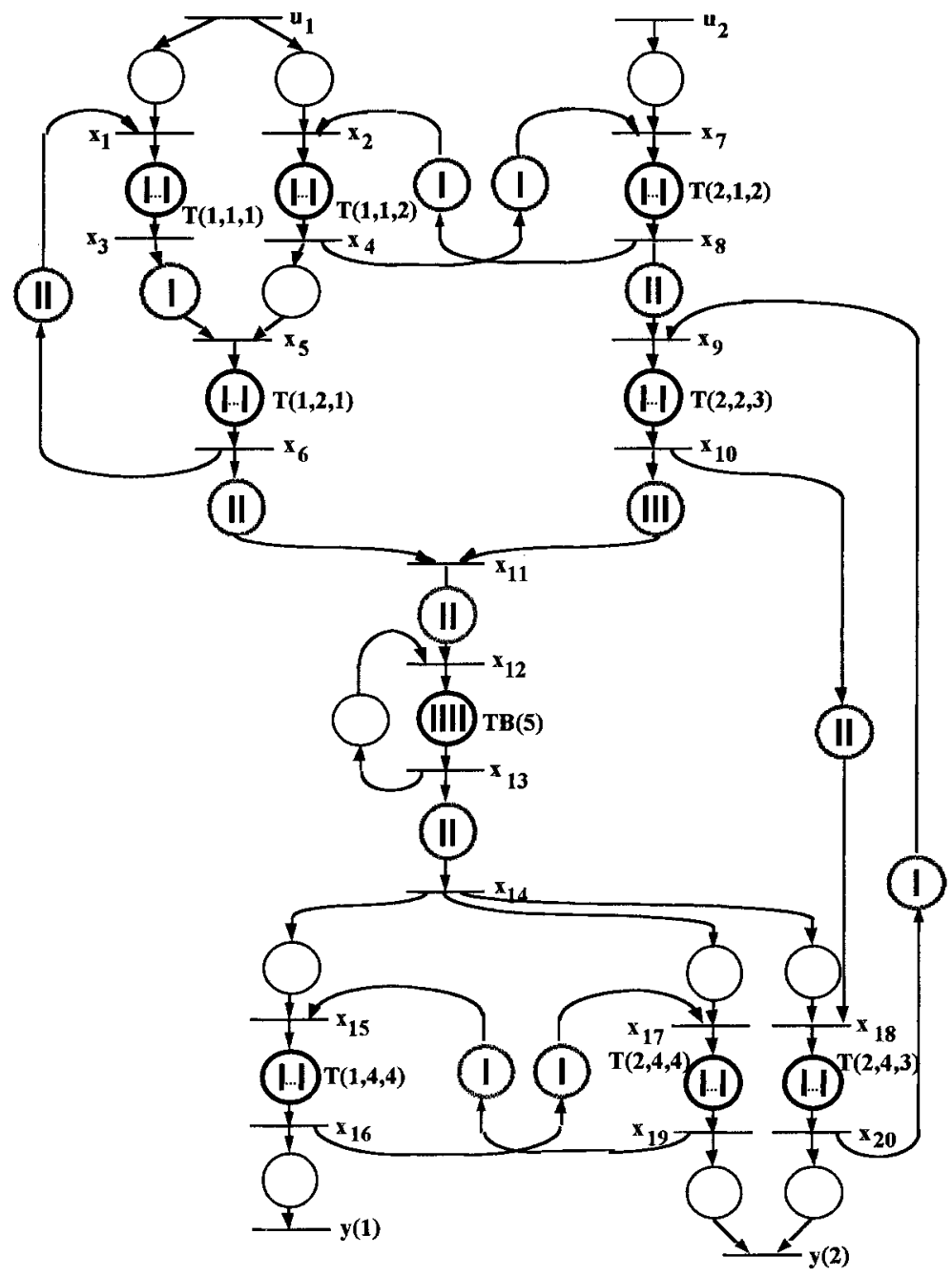

Figure 1

\section{ALGEBRAIC COMPUTATION OF THE PERFORMANCE INDICES}

\subsection{Representation of Timed Event Graphs as linear systems}

A major feature of Timed Event Graphs is that they can be represented through equations formally similar to those of linear time-invariant systems, provided that some tools peculiar to the so-called Max-Plus algebra are used. 
The system representation is obtained in a two-dimensional domain resulting from the juxtaposition of an event domain and a time domain (Cohen et al., 1989). To obtain this kind of representation, the introduction of two classes of variables is required: the first class refers to the dates of event occurrences (daters), whereas the second class refers to the number of events occurred (counters). The use of such an algebraic structure, known as MinMax $<<\gamma, \delta>>$ allows to get, for any event graph, the following equations:

$\mathbf{x}=\mathbf{A} \otimes \mathbf{x} \oplus \mathbf{B} \otimes \mathbf{u}$

$\mathbf{y}=\mathbf{C} \otimes \mathbf{x}$

where:

- $x=\operatorname{col}\left[x_{i}, i=1, . ., n s\right], n s=$ number of intermediate transitions (i.e., transitions which have both input and output places), is the state vector: $\mathrm{x}_{\mathbf{i}}$ represents all the information about the $\mathrm{i}$-th transition;

- $\mathbf{u}=\operatorname{col}\left[\mathrm{u}_{\mathrm{i}}, \mathrm{i}=1, . ., \mathrm{ni}\right]$, ni=number of input transitions (transitions which have only output places), is the input vector: $u_{i}$ represents all the information about the $i$-th input transition;

- $\mathbf{y}=$ col[ $\left.y_{i}, i=1, . ., n o\right]$, no=number of output transitions (transitions which have only input places), is the output vector: $y_{i}$ represents all the information about the $\mathrm{i}$-th output transition;

- matrices $\mathbf{A}(\operatorname{dim}(\mathbf{A})=\mathrm{ns} \times \mathrm{ns}), \mathbf{B}(\operatorname{dim}(\mathbf{B})=\mathrm{ns} \times \mathrm{ni})$, and $\mathbf{C}(\operatorname{dim}(\mathbf{C})=$ no $\times$ ns $)$ represents the relations between intermediate transitions input transitions, and output transitions;

- $\otimes$ and $\oplus$ are the two fundamental operators of the considered algebraic structure.

The elements of matrices $\mathbf{A}, \mathbf{B}$, and $\mathbf{C}$ are written in terms of two shift operators, the first one $(\gamma)$ acting in the event domain, and the second $(\delta)$ acting in the time domain.

It is important to remark that equations (1) depend not only on the TEG topological structure, but also on the numbers of bars representing the holding times of places, and on the initial positions of tokens. Once defined the system matrices, it is possible to determine a transfer matrix for the system, describing the relations between vectors $\mathbf{u}$ and $\mathbf{y}$. The general expression of a transfer matrix is given by

\section{$\mathbf{H}=\mathbf{C A}^{*} \mathbf{B}$}

with $\mathbf{A}^{*}=\mathbf{E} \oplus \oplus_{\mathrm{i}=1}^{+} \mathbf{A}^{\mathbf{i}}$ where $\mathbf{E}$ is the identity matrix in the Max-Plus algebra (Baccelli et al, 1992). Once obtained $\mathbf{H}, \mathbf{y}$ can be computed as $\mathbf{y}=\mathbf{H u}$.

\subsection{Conditions for system stability}

In the following, $u(i)$ will denote the arrival process of the lot(s) relevant to the $i$-th product. Then, one can write $u^{=}=\operatorname{col}[u(i), i=1, ., p]$ where $u(i)=\delta^{\tau(i)}\left(\gamma \delta^{\pi(i)}\right)^{*}$, which represents a sequence of events occurring at a constant rate $1 / \pi(i)$, starting at instant $\tau(i)$. For instance, in the example introduced in Section 2, the input vector is given by

$\mathbf{u}=\operatorname{col}\left[\left(\gamma \delta^{\pi(1)}\right)^{*}, \delta^{\tau(2)}\left(\gamma \delta^{\pi(2)}\right)^{*}\right]$

implicitly assuming $\tau(1)=0$

Since it is assumed that the arrival processes last over an infinite horizon, it is necessary to investigate under which conditions the stability of the system is ensured (the system is stable if the number of tokens in any place of the net remains always limited, i. e., if the net is $\mathrm{k}$ bounded, for some finite $\mathrm{k}$ ). To this end, let us first give the following definition: an irreducible TEG modelling a manufacturing system is a system which cannot be partitioned 
into completely separate subsystems (i.e., subsystems which do not share any machine). Then, define the cycle time $\mathrm{C}(\chi)$ of the elementary circuit $\chi$ as the sum of the holding times of the places making up the elementary circuit $\chi$, divided by the number of tokens in the circuit. It is apparent that $C(\chi)$ is an affine function of the decision variables (more specifically, the lot sizes) for any elementary circuit $\chi$.

Result. An irreducible TEG modelling a manufacturing system is stable if and only if the two following conditions are satisfied:
1. $\pi(\mathrm{i})=\bar{\pi}$
$\mathrm{i}=1, \ldots, \mathrm{p}$
2. $\bar{\pi} \geq \mathrm{C}(\chi)$
$\forall$ elementary circuit $\chi$ in the network

Proof. Let us first demonstrate the necessity. As regards the first condition, let us recall that the irreducibility assumption imposes that no production route is completely disjoint from the rest of the system. Thus, consider product $i$, and suppose that it shares a machine $\mathbf{M}_{h}$ with another product $j$. If we admit $\pi(i)>\pi(j)$, then there will be an accumulation of tokens in the buffer preceding $\mathbf{M}_{\mathbf{h}}$, representing items of product $\mathbf{P}_{\mathbf{j}}$. This proves the necessity of the first condition.

As regards the second condition, suppose that an elementary circuit $\chi$ such as $\bar{\pi}<\mathrm{C}(\chi)$ exists. Then, since $1 / C(\chi)$ is the maximum rate at which any transition in that circuit can fire, and owing to the irreducibility assumption, the number of tokens representing parts waiting for entering circuit $\chi$ is increasing. Thus, also the second condition is necessary.

Then, let us come to the sufficiency part. It can be shown (Cohen et al., 1989; Baccelli et al., 1992; Parodi et al., 1994) that the only terms of the type $\left(\gamma^{\alpha} \delta^{\beta}\right)^{*}$ appearing in matrix $\mathbf{H}$ are those associated with the elementary circuits, and are characterized by a value of $\alpha$ equal the number of tokens and of $\beta$ equal to the cycle time of the considered circuit. On the counterpart, the only * term appearing in vector $\mathbf{u}$ is $\left(\gamma \delta^{\bar{\pi}}\right)^{*}$, if the first condition holds. Due to the simplification rules in the Max-Plus algebra, and to the second condition, the * terms appearing in vector $\mathbf{y}$ are all of the same type, that is, $\gamma \delta^{\bar{\pi}}$. This implies that the output rate for any product $i$ in the steady-state equals the input rate, thus proving the stability of the overall system.

As a consequence of the above first stability condition, all decision variables $\pi(i)$ reduce to a single variable $\bar{\pi}$.

\subsection{Evaluation of the performance indices}

The computation of matrix $\mathbf{A}^{*}$ can be performed following the rules reported in (Baccelli et al., 1992). It is worth remarking that several additional simplification rules have to be applied in the presented model, since matrix $\mathbf{A}^{*}$ has an additional dependence on the components of $\xi$ (besides to $\gamma$ and $\delta$ ). These rules are reported in detail in (Parodi et al., 1994), and are omitted here for brevity. Similar considerations hold for the determination of $\mathbf{H}$.

If the above stability conditions hold, the system is stable, and thus it makes sense to consider its steady-state behaviour. More specifically, the indices of interest will be the throughputs and the steady-state flow times.

In general, the overall throughput of the $\mathrm{i}$-th product, namely $\mathrm{X}(\mathrm{i})$ is given by 


$$
\mathrm{X}_{\mathrm{i}}=\sum_{\mathrm{k} \in M\left(\mathrm{i}, \mathrm{n}_{\mathrm{i}}\right)} \mathrm{N}\left(\mathrm{i}, \mathrm{n}_{\mathrm{i}}, \mathrm{k}\right) / \bar{\pi}
$$

The flow time $\mathrm{ft}(\mathrm{i}, \mathrm{r})$ of token $\mathrm{r}$ for the $\mathrm{i}$-th class of products (this is the $(\mathrm{r}+1)$-th token of product $P_{i}$ that flows in the system, since the first is token 0 ) can be determined as the difference between the exit time instant and the entrance time instant. This requires the determination of the output sequence for the considered product. In the proposed model, it turns out (Parodi et al., 1994) that all output sequences (note that each product has only one output sequence) have the structure

$y(i)=Q(i) \otimes\left(\gamma \delta^{\bar{\pi}}\right)^{*}$

where $Q(i)$ is a polynomial in the operators $\gamma$ and $\delta$, having the structure

$\mathrm{Q}(\mathrm{i})=\bigoplus_{\mathrm{q}=0}^{\mathrm{n}(\mathrm{i})} \gamma \mathrm{q} \delta \delta_{\mathrm{q}}(\mathrm{i})$

where the coefficients $f_{\mathrm{q}}(\mathrm{i}), \mathrm{i}=1, \ldots, \mathrm{p}$, are affine functions (in the conventional algebra) of parameters $\mathrm{N}(1, \mathrm{j}, \mathrm{k})$ and $\tau(1), \forall 1, \mathrm{j}, \mathrm{k}$.

On this basis, it is possible to evaluate the steady-state flow time relevant to product $\mathbf{P}_{\mathbf{i}}$ (Di Febbraro et al., 1994), which turns out to be

$\overline{f t}(i)=\max \left\{f_{0}(i)+n(i) \bar{a}, f_{1}(i)+(n(i)-1) \bar{a}, \ldots, f_{n(i)}(i)\right\}-(n(i) \bar{a}+\tau(i))$

\section{THE OPTIMIZATION PROBLEM}

Having so determined the performance indices of interest, several optimization problems can be stated. In this paper, the problem consisting in the maximization of the weighted system throughput, while keeping the steady-state flow time of each product limited by an a-priori upper bound, is considered.

Problem 1: Maximize the objective function

$$
\max _{\xi} \sum_{\mathrm{i}=1}^{\mathrm{p}} \mathrm{w}_{\mathrm{i}} \sum_{\mathrm{k} \in M\left(\mathrm{i}, \mathrm{n}_{\mathrm{i}}\right)} \mathrm{N}\left(\mathrm{i}, \mathrm{n}_{\mathrm{i}}, \mathrm{k}\right) / \mathbf{a}
$$

subject to

$$
\begin{aligned}
& N_{\min }(i, j, k), N(i, j, k) . N \max (i, j, k) \\
& \bar{\pi} \geq C(\chi) \\
& \bar{f}(i) \leq \overline{\mathbf{F}}_{i}
\end{aligned}
$$

$$
\begin{aligned}
\mathrm{i}= & 1, \ldots, \mathrm{p}, \mathrm{j}=1, . ., \mathrm{n}_{\mathrm{i}}, \mathrm{k} \in M(\mathrm{i}, \mathrm{j}) \\
& \forall \text { elementary circuit } \chi \text { in the network } \\
& \mathrm{i}=1, \ldots, \mathrm{p}
\end{aligned}
$$




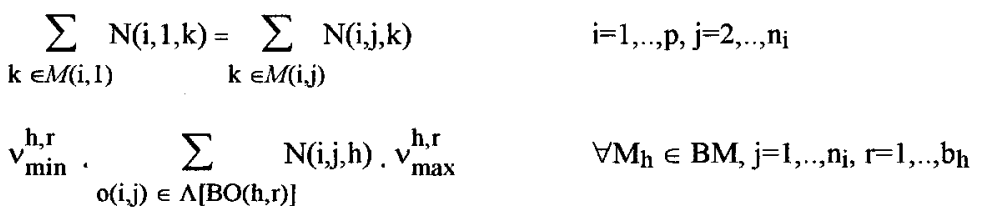

where

- coefficients $\mathrm{w}_{\mathrm{i}}$ are a-priori given constants;

- constraints (8) impose a-priori lower and upper bounds on the lot sizes of components of product class $\mathrm{P}_{\mathrm{i}}$;

- constraints (9) guarantee the fulfilment of the second stability condition;

- in constraints (10), $\overline{\mathrm{F}}_{\mathrm{i}}$ is the a-priori given upper bound for the steady-state flow time of the class of product $\mathbf{P}_{\mathrm{i}}$;

- constraints (11) guarantee the 'flow conservation' of the parts.

Owing to the structure of the terms $\overline{\mathrm{f}}(\mathrm{i})$, as given by (6), to the fact that the terms $\mathrm{f}_{\mathrm{q}}(\mathrm{i})$, as well as the cycle time $\mathbf{C}(\chi)$, are affine functions of the decision variables, Problem 1 turns out to be a linear fractional programming problem which can be solved by means of standard techniques (Bazaraa and Shetty, 1980) and software tools.

The solution of the above optimization problem, with reference to a numerical example corresponding to the net structure depicted in Figure 1, has required a computation time of about 2 hours on a Workstation HP 9000/720, by use of a purposely developed software package based on the commercial code MAPLE $\mathrm{V}$.

Finally, it is worth remarking that assuming to have fixed the initial marking of the net is not too restrictive. In fact, the number of tokens in a machine circuit is constrained to be equal to the number of servers of that machine. Moreover, the tokens in the initial marking representing the parts already present in the system are actually not relevant as regards the steady-state behaviour of the system, provided that the stability conditions are fulfilled.

\section{REFERENCES}

Baccelli, F.L., Cohen, G., Olsder, G.J. and Quadrat, J.P. (1992) Synchronization and Linearity. Wiley, New York.

Bazaraa, M. and Shetty, C. (1980) Nonlinear Programming. Wiley, New York.

Cohen, G., Dubois, D., Quadrat, J. P. and Viot, M. (1985) A linear-system-theoretic view of discrete-event processes and its use for performance evaluation in manufacturing. IEEE Transactions on Automatic Control, 30, 210-220.

Cohen, G., Moller, P., Quadrat, J. P. and Viot, M. (1989) Algebraic tools for the performance evaluation of discrete event systems. Proc. IEEE, 77, 39-58.

Di Febbraro A., Minciardi, R. and Sacone S. (1994) Performance optimization in manufacturing systems by use of Max-Plus algebraic techniques. Proc. IEEE Int. Conference on Systems, Man, and Cybernetics, 2, 1995-2001.

Parodi, G., Prati, A. and Profumo, M. (1994) Metodi algebrici per l'analisi e l'ottimizzazione di sistemi di produzione automatizzata rappresentati tramite Reti di Petri. Tesi di Laurea, Universita di Genova (in Italian). 